\title{
Ringen um Beteiligung und Einfluss: Die Vertretung schwacher Interessen im Sozialstaat
}

\author{
FLORIAN BLANK, SIMONE LEIBER
}

$\mathrm{S}$ eit vielen Jahren wird über Reformen des Sozialstaats, über ihre Voraussetzungen und Wirkungen diskutiert. Die Reformprozesse und ihre Ergebnisse spiegeln Interessenkonstellationen und -konflikte wider und damit auch die Stärke bzw. Schwäche von Akteuren, die für die Durchsetzung spezifischer Interessen stehen. In der öffentlichen Debatte werden Reformen häufig hinsichtlich ihrer Verteilungswirkung bewertet: Welche Akteursgruppe wird durch Reformen sozioökonomisch bessergestellt, welche verliert? Weniger Beachtung finden hingegen Fragen der politischen (Un-)Gleichheit: Welche Interessen werden im sozialpolitischen Reformdiskurs überhaupt gehört und können sich Geltung verschaffen? Und wie wirken gesellschaftliche Veränderungen und Politikergebnisse auf diese Interessen zurück?

Das vorliegende Schwerpunktheft stellt diese zweite Perspektive auf Sozialpolitik ins Zentrum. Es diskutiert die Organisations- und Konfliktfähigkeit sogenannter „schwacher Interessen“ im Politikfeld Sozialpolitik. Wie können Menschen, die soziale Unterstützung in Anspruch nehmen, ihre Wünsche und Forderungen an die Politik artikulieren? Inwiefern können sie ihre Interessen durchsetzen? Diese Fragen stellen sich, weil potenzielle oder tatsächliche Bezieher*innen von Sozialleistungen, aber auch Hilfsbedürftige, für die es noch gar kein Unterstützungsangebot gibt, häufig nur begrenzte Möglichkeiten haben, sich für ihre Interessen einzusetzen. Solche schwachen Interessen sind vergleichsweise schwer zu organisieren, ihnen fehlen wichtige Ressourcen für die Durchsetzung in der Politik. Welche Interessen dabei zu den (vermeintlich) schwachen oder starken Interessen gehören, ist allerdings nicht für immer festgelegt und bedarf einer kontinuierlichen Bestandsaufnahme. Zu dieser will das Schwerpunktheft beitragen und damit eine Debatte wieder aufnehmen, die ihren Höhepunkt in der Politikwissenschaft und politischen Soziologie in Deutschland vor allem in den Jahren zwischen 2000 und 2010 hatte.

Das Heft baut auf einer 2019 an der Universität Duisburg-Essen durchgeführten Tagung der Sektion Sozialpolitik der Deutschen Gesellschaft für Soziologie und des Arbeitskreises Wohlfahrtsstaatsforschung in der Deutschen Vereinigung für Politikwissenschaft auf. An der Konzeption des Heftes haben aus diesen Fachgesellschaften auch Tanja Klenk, Michaela Schulze und Jana Windwehr mitgearbeitet.

Die Berücksichtigung schwacher Interessen in der Sozialpolitik ist aus zwei Gründen wichtig: Einerseits um das Sozialstaatsgebot des Grundgesetzes sowie die Ziele „sozialer Gerechtigkeit und sozialer Sicherheit“ (wie es im Eingangsparagrafen des Sozialgesetzbuches I heißt) zu erfüllen. Andererseits aus Gründen der Demokratie: Die Sozialforschung hat in den vergangenen Jahren herausgearbeitet, dass in Hinblick auf Wahlentscheidungen wie auch auf die politische Responsivität in der deutschen Politik keine Gleichheit herrscht. Eine dauerhafte Nicht-Beachtung schwacher Interessen in Bezug auf materielle Teilhabe und grundlegende Anerkennung kann zu berechtigter Frustration und einer Abwendung vom politischen System oder dem demokratischen Parteienspektrum führen.

Betont werden muss ebenfalls: Es handelt sich hierbei nicht um ein gesellschaftliches Randphänomen. Denn unter die Kategorie „schwache Interessen“ werden auch Gruppen gefasst, die in der Mitte der Gesellschaft stehen. Teils betreffen schwache Interessen sogar die Lebensrealität einer großen Gruppe oder Mehrheit der Bevölkerung: Familien, Patient*innen etwa, aber auch Sozialleistungsempfänger ${ }^{*}$ innen, Beschäftigte im Sozialsektor oder behinderte Menschen zählen mit dazu.

Die in diesem Heft versammelten Beiträge nähern sich dem Thema aus unterschiedlichen Blickwinkeln. Übergreifend sind für alle die Fragen, welche zentralen Veränderungen sich insbesondere in der vergangenen Dekade ergeben haben und wie eine Stärkung schwacher Interessen gelingen kann. Ein wichtiger roten Faden des Heftes ist auch die Frage nach geeigneten Wegen oder Modi der Interessenvertretung: Wieviel Selbstvertretung durch Betroffene ist nicht nur wünschenswert, sondern machbar und erfolgversprechend? Wann ist die anwaltschaftliche Interessenvertretung durch Dritte notwendig, wann bevormundend? Welche Bedeutung hat die Unterstützung durch etablierte Akteure, durch Parteien, Verbände, Organisationen Sozialer Arbeit, gesellschaftliche Initiativen oder engagierte Einzelpersonen - nicht nur, aber auch durch Hilfe zur Selbsthilfe?

Die Schwäche bestimmter Gruppen kann durch staatliches Handeln teilweise ausgeglichen werden. An anderen Stellen scheint dagegen die staatliche Unterstützung zu fehlen oder stößt selbst an Grenzen. Sozialpolitik steht damit vor der Aufgabe, nicht nur an sie gerichtete Forderungen $\mathrm{zu}$ bearbeiten, sondern auch deren Artikulation zu ermöglichen und zu unterstützen. Reformen lassen sich also nicht nur an ihren (Verteilungs-) Wirkungen messen, sondern auch daran, wer im politischen Prozess Gehör fand und wessen Stimme im Ergebnis gestärkt wird.

\section{KONZEPT UND KOORDINATION DES SCHWERPUNKTHEFTES}

FLORIAN BLANK, Dr., Wissenschaftler im Wirtschafts- und Sozialwissenschaftlichen Institut (WSI) der Hans-Böckler-Stiftung. Forschungsschwerpunkt: Sozialpolitik.

\section{florian-blank@boeckler.de}

SIMONE LEIBER, Prof. Dr., Professorin für Politikwissenschaft mit Schwerpunkt Sozialpolitik an der Universität Duisburg-Essen. Forschungsschwerpunkte: Vergleichende Wohlfahrtstaatsforschung, Pflegepolitik, Verhältnis Sozialpolitik und Soziale Arbeit.

simone.leiber@uni-due.de 